\title{
Fatores relacionados as disfunções sexuais femininas durante o puerpério: uma
}

\section{revisão sistemática}

\author{
Factors related to female sexual dysfunction during the postpartum period: a systematic review \\ Factores relacionados con la disfunción sexual femenina durante el puerperio: una revisión
}

sistemática

Recebido: 12/01/2022 | Revisado: 19/01/2022 | Aceito: 21/01/2022 | Publicado: 23/01/2022

\author{
Ana Clara Cunha Parente \\ ORCID: https://orcid.org/0000-0001-9468-5415 \\ Universidade do Estado do Pará, Brasil \\ E-mail: anaclaraparente98@gmail.com \\ Karen Sabriny Costa Regis \\ ORCID: https://orcid.org/0000-0001-8264-2549 \\ Universidade do Estado do Pará, Brasil \\ E-mail: sabrinykaren15@gmail.com \\ Daniely Leal da Costa \\ ORCID: https://orcid.org/0000-0002-2795-2832 \\ Universidade do Estado do Pará, Brasil \\ E-mail: contatodanielyleal@gmail.com
}

\begin{abstract}
Resumo
O puerpério corresponde ao período entre a expulsão do feto e termina quando cessa o estado involutivo dos fenômenos gerados pela gravidez. Algumas complicações independem da via de parto, dentre os principais achados, encontra-se a disfunção sexual, exemplificada, principalmente, pela diminuição do desejo sexual e, consequentemente, diminuição da qualidade de vida da mulher. O objetivo do presente estudo foi analisar quais os principais fatores relacionados as disfunções sexuais femininas durante o puerpério. Trata-se de uma revisão sistemática utilizando do protocolo PRISMA. Sendo assim, a estratégia utilizada neste estudo compreendeu a efetivação de buscas sistemáticas na literatura, a partir das bases de dados PubMed, Lilacs, Scielo e PEDro. Para análise, foram incluídos na pesquisa 16 artigos, onde 2 discutiam sobre as causas psicossociais das disfunções sexuais, 8 salientaram a relação da via de parto com disfunções sexuais, 5 artigos discutiram a relação entre as lacerações perineais com as disfunções sexuais e 3 relataram intervenções fisioterapêuticas no tratamento das disfunções sexuais. Conclui-se que as principais causas das disfunções sexuais foram a ansiedade e depressão, principalmente no puerpério imediato, onde se observou uma necessidade de compreensão do parceiro para a retomada das atividades sexuais. A literatura não apresenta consenso sobre qual via de parto sugere mais disfunções sexuais, porém o histórico de lesão obstétrica pélvica atrapalha o retorno das atividades sexuais, gerando mais dor e fadiga durante o coito. Por sua vez, a via de parto cesárea diminuiu a lubrificação vaginal e a satisfação sexual nas mulheres estudadas.
\end{abstract}

Palavras-chave: Puerpério; Saúde sexual; Fisioterapia.

\begin{abstract}
The postpartum period corresponds to the period between the expulsion of the fetus and ends of the phenomena generated by pregnancy ceases. Some complications are independent of the mode of delivery, among the main findings is sexual dysfunction, exemplified mainly by the decrease in sexual desire and, consequently, a decrease in the woman's quality of life. The objective of the present study was to analyze the main factors related to female sexual dysfunctions during the postpartum period. This is a systematic review using the PRISMA protocol. Therefore, the strategy used in this study included carrying out systematic searches in the literature, using PubMed, Lilacs, Scielo and PEDro databases. For analysis, 16 articles were included in the research. It is concluded that the main causes of sexual dysfunctions were anxiety and depression, especially in the immediate postpartum period, where there was a need for understanding from the partner for the resumption of sexual activities. The literature does not present a consensus on which mode of delivery suggests more sexual dysfunctions, but the history of obstetric pelvic injury hinders the return of sexual activities, generating more pain and fatigue during intercourse. In turn, the cesarean delivery method decreased vaginal lubrication and sexual satisfaction in the women studied.
\end{abstract}

Keywords: Postpartum period; Sexual health; Physiotherapy. 


\begin{abstract}
Resumen
El puerperio corresponde al período comprendido entre la expulsión del feto y termina cuando cesa el estado involutivo de los fenómenos generados por el embarazo. Algunas complicaciones son independientes de la modalidad del parto, entre los principales hallazgos está la disfunción sexual, ejemplificada principalmente por la disminución del deseo sexual y disminución de la calidad de vida de la mujer. El objetivo del presente estudio fue analizar los principales factores relacionados con las disfunciones sexuales femeninas durante el puerperio. Esta es una revisión sistemática utilizando el protocolo PRISMA. Por lo tanto, la estrategia utilizada en este estudio incluyó la realización de búsquedas sistemáticas en la literatura, utilizando las bases de datos PubMed, Lilacs, Scielo y PEDro. Para el análisis, se incluyeron 16 artículos en la investigación. Se concluye que las principales causas de las disfunciones sexuales fueron la ansiedad y la depresión, especialmente en el puerperio inmediato, donde hubo necesidad de comprensión por parte del compañero para la reanudación de las actividades sexuales. La literatura no presenta un consenso sobre qué modalidad de parto sugiere más disfunciones sexuales, pero el antecedente de lesión pélvica obstétrica dificulta el retorno a las actividades sexuales, generando más dolor y fatiga durante el coito. A su vez, el método de parto por cesárea disminuyó la lubricación vaginal y la satisfacción sexual en las mujeres estudiadas.
\end{abstract}

Palabras clave: Periodo posparto; Salud sexual; Fisioterapia.

\title{
1. Introdução
}

O puerpério corresponde ao período entre a expulsão do feto e termina quando cessa o estado involutivo dos fenômenos gerados pela gravidez. É dividido em três estágios, independente da via de parto: pós-parto imediato, que vai do $1^{\circ}$ ao $10^{\circ}$ dia após o parto; pós-parto tardio, que corresponde do $11^{\circ}$ ao $40^{\circ}$ dia após o parto; e pós-parto remoto, do $41^{\circ}$ dia em diante (Girardi, 2019).

Segundo dados disponibilizados no DATASUS, na cidade de Santarém-Pará, a via de parto cesárea entre os anos de 1999 e 2008 obteve um aumento de 15,8 \% para 20,9\%. Esta elevação no índice se relaciona, diretamente, ao maior risco de infecção pós-parto, infecção urinária, cefaleia, dor e complicações da anestesia; e, diferentemente do esperado, não é fator de proteção contra complicações tardias, como incontinência urinária e fecal, cistocele e prolapso de útero. As mulheres submetidas à cesariana também apresentaram 2,40 vezes mais relatos de dor em comparação às mulheres que tiveram parto vaginal. Outra pesquisa realizada no Brasil, mostrou que mulheres que tiveram parto vaginal apresentavam $82 \%$ menor chance de dor intensa no pós-parto (Cardoso et al., 2010; Datasus, 2009; Mascarello, 2018).

Além das complicações devido a via de parto, outras surgem durante este ciclo da vida, como demonstra o estudo de Pereira et al., (2018), no qual 91\% de uma amostra entrevistada durante o puerpério remoto apresentaram algum tipo de queixa. Nesse contexto, dentre os principais achados, encontra-se a disfunção sexual, exemplificada, principalmente, pela diminuição do desejo sexual e, consequentemente, diminuição da qualidade de vida da mulher.

Por sua vez, algumas pesquisas comprovam os benefícios da fisioterapia em todos os níveis de atenção à saúde da mulher. Na atenção primária, o fisioterapeuta utiliza estratégias de educação em saúde no pós-parto imediato, conduzidas com utilização de materiais de apoio simples e de baixo custo. A nível ambulatorial, existem diversos recursos e técnicas nãofarmacológicas para diminuição das dores e prevenção de disfunções através da Eletroestimulação Nervosa Transcutânea (TENS), crioterapia, treinamento dos músculos de assoalho pélvico (TMAP) e reeducação abdominal (Da Silva et al.,2019; Santana et al., 2011).

Diante desse contexto, o objetivo do presente estudo foi analisar quais os principais fatores relacionados as disfunç̃es sexuais femininas durante o puerpério.

\section{Metodologia}

Trata-se de uma revisão sistemática utilizando do protocolo PRISMA (Galvão e Ricarte et al.,2019). Sendo assim, a estratégia utilizada neste estudo compreendeu a efetivação de buscas sistemáticas na literatura, a partir das bases de dados PubMed, Lilacs, Scielo e PEDro. Não foram utilizados filtros por desenho de estudo, tamanho da amostra e sem período de 
cobertura do estudo, sendo assim incluindo todos os anos. Durante a revisão sistemática, foram utilizadas palavras incluídas nos recursos dos Descritores em Ciências da Saúde (DeCS). As combinações de descritores resultaram nos seguintes termos: Período Pós-Parto, Fisioterapia e Saúde Sexual. Além disso, foram aplicados os operadores booleanos "OR" e "AND". Os descritores foram escritos na língua inglesa para ampliar o potencial de busca das publicações.

Durante a revisão sistemática, foram considerados como critérios de inclusão: estudos publicados nos idiomas português, inglês ou espanhol disponíveis através de texto completo; que obtivessem objetivo do estudo relacionado ao puerpério e com alguma intervenção fisioterapêutica. Critérios de exclusão: teses, dissertações, monografias não publicadas e temas não relacionados com o objetivo do estudo.

Primeiramente, foram realizadas as buscas em cada base de dados, e após leitura do título, foram excluídos os artigos duplicados. A partir de então, foi realizada a leitura dos resumos, onde, nesta etapa, se excluíram os artigos incompletos ou que não se encaixavam ao tema proposto. Finalmente, os artigos foram baixados e passaram pela leitura na íntegra para alocação nas categorias com o objetivo de enriquecer a discussão dos autores. Ademais, todos os dados alimentaram tabelas do programa Microsoft Excel.

Para desenho das categorias, foi levado em consideração o conteúdo do artigo e sua relação com o tema disfunção sexual, onde obteve-se: Causas psicossociais e disfunção sexual (DS); Tipo de parto e DS; DS e Laceração ou Trauma Perineal; e Intervenção Fisioterapêutica nas DS. Vale ressaltar que alguns artigos possuíam conteúdo que se enquadravam em mais de uma categoria.

Após a categorização dos artigos incluídos, foram extraídos, de forma independente, as informações necessárias para fomentação da tabela final, obedecendo um check list composto pelos itens: periódico, autores e ano; objetivo do estudo; amostra; tipo de estudo; métodos; e resultados. Por fim, os dados foram comparados e discutidos.

A Figura 1 mostra o fluxograma baseado no método PRISMA sobre o protocolo utilizado na pesquisa realizada. 
Research, Society and Development, v. 11, n. 2, e23111225638, 2022

(CC BY 4.0) | ISSN 2525-3409 | DOI: http://dx.doi.org/10.33448/rsd-v11i2.25638

Figura 1. Fluxograma baseado no método PRISMA sobre o protocolo utilizado na pesquisa realizada.
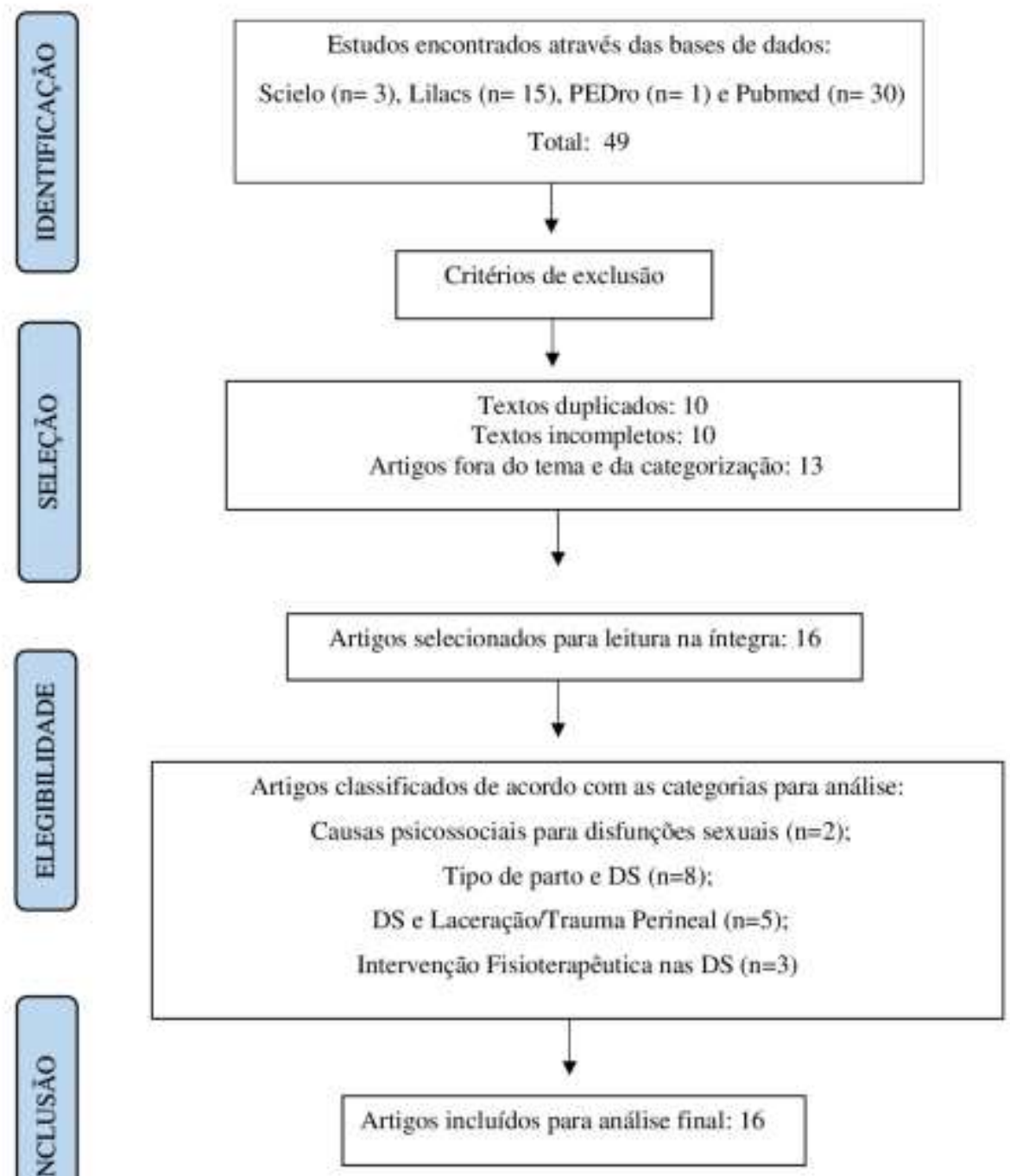

Fonte: Autores.

\section{Resultados}

Para análise, foram incluídos na pesquisa 16 artigos, onde 2 discutiam sobre as causas psicossociais das disfunções sexuais, 08 salientaram a relação da via de parto com disfunções sexuais, 05 artigos discutiram as lacerações perineais com as disfunções sexuais e 03 relataram intervenções fisioterapêuticas no tratamento das disfunções sexuais. Portanto, 16 artigos foram inclusos na pesquisa. O Quadro 1, a seguir, demonstra as características dos estudos após a análise dos dados. 
Quadro 1. Relação dos artigos sobre disfunções sexuais no puerpério.

\begin{tabular}{|c|c|c|c|c|c|}
\hline $\begin{array}{c}\text { PERIÓDICO/CIT } \\
\text { AÇÃ̃ } \\
\end{array}$ & OBJETIVO & $\begin{array}{l}\text { AMOSTRA } \\
\text { (n) }\end{array}$ & $\begin{array}{l}\text { TIPO DE } \\
\text { ESTUDO }\end{array}$ & MÉTODO & RESULTADOS \\
\hline $\begin{array}{c}\text { Pubmed/ } \\
\text { Faisal-Cury et al. } \\
2013\end{array}$ & $\begin{array}{l}\text { Avaliar a relação } \\
\text { entre sintomas } \\
\text { depressivos durante } \\
\text { o período perinatal e } \\
\text { vida sexual pós- } \\
\text { parto. }\end{array}$ & 644 & $\begin{array}{c}\text { Coorte } \\
\text { prospectivo }\end{array}$ & $\begin{array}{l}\text { Entrevistas com mulheres entre } \\
20 \text { e } 30 \text { semanas de } \\
\text { gravidez e depois no pós-parto } \\
\text { utilizando o questionário sobre } \\
\text { padrões sexuais } \\
\text { e o SRQ-20. }\end{array}$ & $\begin{array}{l}\text { Declínio da vida sexual (DVS) ocorreu em } \\
21,1 \% \text {. Variáveis associadas: sintomas } \\
\text { depressivos / ansiosos durante a gravidez e o } \\
\text { pós-parto (RR: } 3,17 \text { [IC 95\%: } 2,18-4,59] \text { ]) }\end{array}$ \\
\hline $\begin{array}{c}\text { Lilacs/ } \\
\text { Chaparro, Perez e } \\
\text { Saez } 2013\end{array}$ & $\begin{array}{l}\text { Analisar as variáveis } \\
\text { biopsicossociais } \\
\text { associadas à função } \\
\text { sexual feminina no } \\
\text { período pós-parto }\end{array}$ & 117 & $\begin{array}{l}\text { Analítico, } \\
\text { transversal e } \\
\text { correlacional }\end{array}$ & $\begin{array}{c}\text { Entrevista utilizando } \\
\text { instrumento para obter dados } \\
\text { sociodemográficos, saúde } \\
\text { sexual e reprodutiva pós-parto e } \\
\text { o Índice de Função Sexual } \\
\text { Feminina (FSFI). }\end{array}$ & $\begin{array}{c}\text { FSFI médio foi de } 22,1 \text { pontos. } 73,6 \% \text { das } \\
\text { mulheres apresentavam DS. FSFI/ desejo e } \\
\text { excitação era maior nas mulheres que } \\
\text { começaram atividade sexual porque queriam, } \\
\text { do que naquelas que } \\
\text { começou porque seu parceiro insistiu. Orgasmo } \\
\text { domínio com a maior porcentagem para } \\
\text { DS }(83 \%) \text {. }\end{array}$ \\
\hline $\begin{array}{c}\text { Pubmed/ } \\
\text { Baud et al. } 2020\end{array}$ & $\begin{array}{l}\text { Avaliar sintomas } \\
\text { fecais, urinários e } \\
\text { sexuais } 6 \text { anos após } \\
\text { o parto, comparando } \\
\text { o parto vaginal não } \\
\text { complicado e o } \\
\text { parto cesáreo } \\
\text { eletivo. }\end{array}$ & $\begin{array}{l}309 \text { com partos } \\
\text { vaginais únicos } \\
\text { não } \\
\text { complicados e } \\
208 \text { com partos } \\
\text { cesáreos } \\
\text { eletivos únicos }\end{array}$ & Transversal & $\begin{array}{l}\text { Quatro questionários validados } \\
\text { autoadministrados: as formas } \\
\text { curtas do UDI-6, IIQ-7, Escala } \\
\text { de Wexner e FSFI. }\end{array}$ & $\begin{array}{c}\text { Mulheres de PCEU relataram mais dificuldades } \\
\text { na lubrificação e satisfação, do que as de PVSC. } \\
\text { Dor durante ou após a relação sexual foram } \\
\text { piores PCEU. Dispareunia mais frequentes } \\
\text { PCEU do que PVSC. }\end{array}$ \\
\hline $\begin{array}{c}\text { Pubmed/ } \\
\text { Fan et al. } 2017\end{array}$ & $\begin{array}{l}\text { Explorar o modo de } \\
\quad \text { parto e } \\
\text { diferenças na função } \\
\text { sexual pós-parto } \\
\text { (curto e } \\
\text { longo prazo) em } \\
\text { mulheres primíparas } \\
\text { chinesas } \\
\end{array}$ & $\begin{array}{l}10 \text { estudos com } \\
\text { uma população } \\
\text { total de } 2.851\end{array}$ & $\begin{array}{l}\text { Revisão } \\
\text { sistemática e } \\
\text { meta-análise }\end{array}$ & $\begin{array}{l}\text { Pesquisas eletrônicas realizadas } \\
\text { para identificar qualquer estudo } \\
\text { em bancos de dados publicado } \\
\text { até } 31 \text { de agosto de } 2017 \text {. }\end{array}$ & $\begin{array}{l}\text { Via de parto cesárea e parto vaginal espontânea } \\
\text { não afetou a satisfação sexual pós-parto e } \\
\text { pareceu ter efeito mínimo sobre as relações } \\
\text { sexuais retomadas de longo prazo e a dor sexual } \\
\text { em primíparas chinesas. }\end{array}$ \\
\hline $\begin{array}{c}\text { Pubmed/ } \\
\text { Dean et al. } 2008\end{array}$ & $\begin{array}{l}\text { Relação da função } \\
\text { sexual com a } \\
\text { história do modo de } \\
\text { parto, exercícios } \\
\text { para músculos do } \\
\text { assoalho pélvico } \\
\text { (PFMEs) e } \\
\text { incontinência. }\end{array}$ & 2.765 & Transversal & $\begin{array}{l}\text { Questionário postal } 3 \text { meses } \\
\text { pós-parto sobre a prevalência } \\
\text { de incontinência urinária e } \\
\text { fecal. Um } 2^{\circ} \text { questionário foi } \\
\text { enviado após } 6 \text { anos para todas } \\
\text { as que responderam ao } \\
\text { questionário original, } \\
\text { verificando sintomas } \\
\text { posteriores e FS. }\end{array}$ & $\begin{array}{c}\text { Parto exclusivamente cesariana obteve } \\
\text { pontuação melhor nas questões relacionadas à } \\
\text { percepção do tônus vaginal e satisfação sexual } \\
\text { do parceiro. Mulheres que relataram estar } \\
\text { realizando PFME no momento pontuaram } \\
\text { melhor em } 7 \text { questões. Mulheres com } \\
\text { incontinência urinária ou fecal pontuaram } \\
\text { menos em nas questões de FS. }\end{array}$ \\
\hline $\begin{array}{c}\text { Pubmed/ } \\
\text { Amiri et al. } 2017\end{array}$ & $\begin{array}{l}\text { Comparar os } \\
\text { resultados sexuais } \\
\text { após PV e cesariana }\end{array}$ & 203 mulheres & $\begin{array}{l}\text { Transversal } \\
\text { prospectivo }\end{array}$ & $\begin{array}{l}\text { Questionário composto por } 2 \\
\text { partes: } 1^{\mathrm{a}} \text {-características } \\
\text { sociodemográficas, histórico } \\
\text { obstétrico/ ginecológico e } \\
\text { amamentação; } 2^{\mathrm{a}} \text { - FSFI. }\end{array}$ & $\begin{array}{c}\text { A FS não diferiu entre os } 2 \text { grupos de PV e } \\
\text { cesariana em relação ao nível educacional, } \\
\text { métodos contraceptivos e ocupacional. Tempo } \\
\text { de retomada da atividade sexual foi } 8,9 \pm 1,3 \text {, e } \\
\text { não houve conflito entre os } 2 \text { grupos. A } \\
\text { frequência média de RS no pós-parto foi de } 1,8 \\
\pm 1,2 \text { vezes por semana. }\end{array}$ \\
\hline $\begin{array}{c}\text { Pubmed/ } \\
\text { Anglès-Acedo et al. } \\
2019\end{array}$ & $\begin{array}{c}\text { Comparar a } \\
\text { retomada da RS e } \\
\text { variáveis que } \\
\text { influenciam esta } \\
\text { atividade após o } \\
\text { parto em mulheres } \\
\text { com e sem história } \\
\text { de lesão obstétrica } \\
\text { do esfíncter anal de } \\
\text { acordo com o tipo } \\
\text { de parto. } \\
\end{array}$ & 318 & $\begin{array}{l}\text { Prospectivo, } \\
\text { observacional, } \\
\text { de caso-controle }\end{array}$ & $\begin{array}{l}\text { Dados demográficos, obstétrico } \\
\text { e uroginecológicos foram } \\
\text { coletados. Além da aplicação } \\
\text { do PISQ-12. }\end{array}$ & $\begin{array}{c}\text { Após parto espontâneo, os pacientes sem lesões } \\
\text { apresentaram porcentagem maior de retomada } \\
\text { do coito do que com lesões (98\% vs. 77\%), e } \\
\text { escore PISQ-12 foi maior. PISQ-12 foi melhor } \\
\text { em } \\
\text { mulheres com parto espontâneo em comparação } \\
\text { com aquelas com PVO }\end{array}$ \\
\hline $\begin{array}{c}\text { Pubmed/ } \\
\text { Hjorth et al. } 2019\end{array}$ & $\begin{array}{l}\text { Investigar a relação } \\
\text { entre o tipo de parto } \\
\text { e a saúde sexual de } \\
\text { longo prazo da } \\
\text { mulher }\end{array}$ & $\begin{array}{c}43639 \\
\text { mulheres }\end{array}$ & coorte & $\begin{array}{c}\text { A primeira entrevista realizada } \\
\text { na } 16^{\mathrm{a}} \text { semana de gestação com } \\
\text { informações sobre saúde, estilo } \\
\text { de vida e fatores sócio- } \\
\text { ocupacionais. Entre } 2013 \text { e } \\
\text { 2014, os participantes foram } \\
\text { convidados a responder a um } \\
\text { questionário sobre saúde física, } \\
\text { mental e sexual. }\end{array}$ & $\begin{array}{l}\text { Problemas sexuais nas mulheres com PVE foi } \\
\text { de } 37 \% \text {. Mulheres de cesarianas relataram mais } \\
\text { problemas. Mulheres PVE subsequente a uma } \\
\text { cesariana, e mulheres apenas com PV que } \\
\text { tiveram um ou mais PVs instrumentais, as } \\
\text { chances de problemas sexuais não diferiram das } \\
\text { mulheres com apenas PVE. }\end{array}$ \\
\hline $\begin{array}{c}\text { Scielo e } \\
\text { Lilacs/Pereira et al. } \\
2018\end{array}$ & $\begin{array}{c}\text { Avaliar a FS } \\
\text { feminina no } \\
\text { puerpério remoto em } \\
\text { mulheres } \\
\text { brasileiras e } \\
\text { comparar a DF } \\
\end{array}$ & 78 & Transversal & $\begin{array}{c}\text { A FS das } \\
\text { participantes foi avaliada online } \\
\text { através da versão brasileira do } \\
\text { FSFI entre } 45 \text { e } 180 \text { dias } \\
\text { após o parto. }\end{array}$ & $\begin{array}{l}78 \%(\mathrm{n}=61) \text { apresentaram DS no pós-parto } \\
\text { remoto. Escore médio do FSFI foi de } 22,17 \\
\text { para o pós-parto vaginal e } 21,12 \text { para cesárea. }\end{array}$ \\
\hline
\end{tabular}




\begin{tabular}{|c|c|c|c|c|c|}
\hline & $\begin{array}{c}\text { feminina em relação } \\
\text { a via de parto. }\end{array}$ & & & & \\
\hline $\begin{array}{c}\text { Pubmed/Lawrence } \\
\text { et al. } 2016\end{array}$ & $\begin{array}{l}\text { Efeito das } \\
\text { lacerações perineais } \\
\text { no AP, incluindo } \\
\text { incontinência } \\
\text { urinária/anal, FS e } \\
\text { dor perineal em } \\
\text { nulíparas }\end{array}$ & 448 & $\begin{array}{l}\text { coorte } \\
\text { prospectivo }\end{array}$ & $\begin{array}{c}\text { Sintomas do AP foram } \\
\text { avaliados com questionários } \\
\text { validados e exame físico na } \\
\text { gravidez e } 6 \text { meses pós-parto. } 2 \\
\text { grupos de trauma foram } \\
\text { comparados, aqueles com } \\
\text { períneo intacto ou lacerações de } \\
1^{\circ} \text { grau e aqueles com } \\
\text { lacerações de } 2^{\circ}, 3^{\circ} \text { ou } 4^{\circ} \text { graus. }\end{array}$ & $\begin{array}{c}\text { Trauma perineal não foi associado a } \\
\text { incontinência urinária ou fecal, diminuição da } \\
\text { atividade sexual, dor perineal ou prolapso de } \\
\text { órgão pélvico. Mulheres com trauma tiveram } \\
\text { taxas semelhantes de atividade sexual, no } \\
\text { entanto, tiveram escores de FS ligeiramente } \\
\text { mais baixos ( } 27,3 \text { vs. } 29,1, \mathrm{p}=0,01)\end{array}$ \\
\hline $\begin{array}{c}\text { Pubmed/ } \\
\text { O'Malley et al. } \\
2018\end{array}$ & $\begin{array}{l}\text { Prevalência e fatores } \\
\text { de risco potenciais } \\
\text { para problemas de } \\
\text { saúde sexual aos } 6 \mathrm{e} \\
12 \text { meses pós-parto }\end{array}$ & 832 & $\begin{array}{c}\text { coorte } \\
\text { prospectivo } \\
\text { longitudinal }\end{array}$ & $\begin{array}{l}\text { Coleta de informações sobre } \\
\text { questões de saúde sexual e } \\
\text { dados demográficos das } \\
\text { mulheres. Informações } \\
\text { específicas relacionadas à } \\
\text { saúde sexual e dados } \\
\text { obstétricos. }\end{array}$ & $\begin{array}{c}\text { 46,3\% relataram falta de interesse na atividade } \\
\text { sexual, } 43 \% \text { falta de lubrificação vaginal e } \\
37,5 \% \text { tiveram dispareunia } 6 \text { meses pós-parto. } \\
\text { Rupturas perineais de } 2^{\circ} \text { grau, } 3^{\circ} \text { grau e } \\
\text { episiotomia foram associadas a dispareunia } 6 \\
\text { meses após o parto, mas, dessas somente } \\
\text { lacerações de } 3^{\circ} \text { grau, em associação com } \\
\text { amamentação e dispareunia pré-existente. }\end{array}$ \\
\hline \begin{tabular}{c|} 
Pubmed e \\
PEDro/Hadizadeh- \\
Talasaz, Sadeghi e \\
Khadivzadeh. 2019
\end{tabular} & $\begin{array}{c}\text { Estudos que } \\
\text { determinam o efeito } \\
\text { do exercício do AP } \\
\text { na FS e na qualidade } \\
\text { de vida no pós- } \\
\text { parto. } \\
\end{array}$ & 12 artigos & $\begin{array}{c}\text { Revisão } \\
\text { Sistemática }\end{array}$ & $\begin{array}{c}\text { Pesquisados artigos em persa e } \\
\text { inglês publicados em bancos de } \\
\text { dados. Processo de busca e } \\
\text { seleção de artigos foi } \\
\text { direcionado pela diretriz } \\
\text { (PRISMA) }\end{array}$ & $\begin{array}{l}\text { Evidências mostraram que o treinamento da } \\
\text { musculatura do AP em mulheres primíparas ou } \\
\text { multíparas pode aumentar a função sexual e a } \\
\text { qualidade de vida no pós-parto. }\end{array}$ \\
\hline $\begin{array}{l}\text { Pubmed/Huang et } \\
\text { al. } 2019\end{array}$ & $\begin{array}{l}\text { Efeito da radiação } \\
\text { infravermelha na dor } \\
\text { perineal pós-parto e } \\
\text { FS em primíparas } \\
\text { submetidas a } \\
\text { episiotomia e } \\
\text { laceração perineal } \\
\text { de } 2^{\circ} \text { grau. }\end{array}$ & 78 & $\begin{array}{l}\text { Prospectivo } \\
\text { Randomizado }\end{array}$ & $\begin{array}{l}\text { Primíparas com gravidez a } \\
\text { termo e PVs com episiotomia e } \\
\text { laceração perineal de } 2^{\circ} \text { grau } \\
\text { foram convidadas a participar e } \\
\text { aleatoriamente designadas para } \\
\text { um grupo de infravermelho } \\
\text { distante (FIR) e um grupo } \\
\text { controle. }\end{array}$ & $\begin{array}{c}\text { Maioria teve dor perineal leve uma semana após } \\
\text { o parto }(1,1 \pm 0,9 \text { no grupo controle vs } 1,4 \pm 1,5 \\
\text { no grupo FIR) sem diferença significativa entre } \\
\text { os grupos. Grupo FIR teve uma pontuação total } \\
\text { PISQ-12 mais alta em } 3 \text { meses }(35,4 \pm 6,4 \text { vs } \\
34,7 \pm 5,7) \text { e } 6 \text { meses }(36,4 \pm 5,6 \text { vs } 35,6 \pm 5,7) \\
\text { pós-parto em comparação com o grupo } \\
\text { controle, mas sem diferença estatisticamente } \\
\text { significativa. }\end{array}$ \\
\hline
\end{tabular}

SRQ-20 - Self Report Questionnaire; RR - Crude and adjusted risk ratios; DVS - Declínio da vida sexual; FSFI - Índice de Função Sexual Feminina; DS - Disfunção Sexual; FS - Função sexual; PCEU - parto cesáreo eletivo único; PVSC - partos vaginais sem complicações; UDI-6 - Urogenital Distress Inventory; IIQ-7 Incontinence Impact Questionaire; PFMEs - exercícios para músculos do assoalho pélvico; PV - parto vaginal; PVO - parto vaginal operatório; PVE - parto vaginal espontâneo; RS -Relações Sexuais; PISQ-12 - Questionário Sexual de Prolapso de Órgão Pélvico / Incontinência Urinária-12; AP - Assoalho Pélvico; FIR Infravermelho Distante.

Fonte: Autores (2021). 


\section{Discussão}

\section{- Causas psicossociais das DS no pós-parto}

No estudo de Chaparro, Perez e Saez 2013, realizado no Chile, que utilizou uma amostra de 53 mulheres ativas sexualmente, das quais $50,9 \%$ tiveram parto cesáreo e 49,1\% via vaginal. O início da atividade sexual porque o parceiro insistiu foi de 30,2\% e 69,8\% porque ela queria, 56,6\% indicaram que a primeira relação sexual pós parto foi insatisfatória. No puerpério, foi constatado que 73,6\% das mulheres apresentavam disfunção sexual e o índice de função sexual feminina era maior nas mulheres que começaram a atividade sexual porque queriam fazê-lo, do que naquelas que começaram porque seu parceiro insistiu. Orgasmo foi o domínio com a maior porcentagem (83\%) para disfunção sexual. O desejo sexual diminuiu, ligeiramente, em mulheres que amamentavam exclusivamente. $\mathrm{O}$ desejo e excitação foi maior em mulheres que queriam fazer sexo, em comparação com aqueles que iniciaram a relação sexual porque o parceiro insistiu concluindo que a mulher na fase pós-parto apresenta disfunção sexual relacionada a fatores fisiológicos e emocionais.

Em um panorama nacional, o estudo de Faisal-Cury et al. 2013, corroborou com o estudo de Chaparro demonstrando também um aumento na disfunção sexual relacionado ao puerpério. Nesse estudo, foram incluídas 644 mulheres que haviam retomado a atividade sexual no período pós-parto. $1 \%$ puérperas fizeram uso de antidepressivos, $21,1 \%$ participantes foram classificados como tendo declínio na vida sexual, 45,0\% retomaram a vida sexual durante o primeiro mês após o parto, e menos de $4 \%$ tomaram mais de 6 meses para retomar a relação sexual. Na análise multivariável as pontuações para: episiotomia, parto com fórceps, gestações anteriores, estado de casamento, depressão durante a gravidez, depressão durante apenas o pós-parto, um aborto anterior e a idade do paciente foram significativamente associadas ao declínio sexual (DVS), ocorrendo em $21,1 \%$ da coorte. Contudo, algumas variáveis foram associadas, como: sintomas depressivos e/ou ansiosos durante a gravidez e no período pós-parto. Concluiu-se que esforços para melhorar as taxas de tratamento da depressão ou ansiedade perinatal em ambientes de cuidados primários têm o potencial de preservar o funcionamento sexual para mães de baixa renda.

\section{- A relação entre o tipo de parto e DS no pós-parto}

Segundo Pereira et al. 2018, realizado no Brasil, entre as mulheres incluídas, 30 passaram por parto vaginal e 48 por cesárea. Entre as participantes, 91\% apresentaram algum tipo de queixa no puerpério. Destas, 59,2\% eram de puerpério de cesárea enquanto 40,8\% estavam no puerpério de parto vaginal. Trauma perineal foi relatado por 53,3\% das participantes de puerpério de parto vaginal. Todas as participantes relataram ter retornado à atividade sexual. O tempo médio de retorno às atividades sexuais foi de 52,83 dias após o parto vaginal e 52,94 dias após cesárea. A disfunção sexual esteve presente em $78,2 \%$ dos participantes do estudo, $78 \%$ apresentaram disfunção sexual no pós-parto remoto, sendo que o escore médio do FSFI foi de 22,17 para o pós-parto vaginal e 21,12 para o puerpério de cesárea, a maior parte das mulheres no puerpério remoto apresentou disfunção sexual. Por seguinte, não foi encontrada diferença significativa na função sexual feminina entre os tipos de parto.

Moura et al. 2018 corroboraram com o estudo de Pereira et al. 2018, no qual foram encontrados 28 artigos relacionados a dispareunia (dor genital associada a relação sexual) pode ocorrer em 24,0 a 85,7\% das puérperas. Após o primeiro parto vaginal, 21,0\% apresentam avulsão dos levantadores do ânus, mas 62,0\% não são evidentes após um ano. A alteração do corpo perineal não está relacionada a laceração ou a função sexual, mas 32,5\% das mulheres que relataram dor perineal no primeiro mês relataram dispareunia aos 6 meses. 85,7\% relatam dor na primeira relação pós-parto e as que tiveram cesariana foram mais propensas a dispareunia seis meses após o parto. 31,5\% das lactantes aos 6 meses e 24,1 a 28,3\% das que apresentaram queixas psicossociais relataram dispareunia. A literatura não apresenta consenso sobre qual via de parto pode levar a maior potencial de disfunção sexual a curto, médio e longo prazo, evidenciando que tanto o parto vaginal, sendo 
instrumentalizado ou não, quanto a cesárea, eletiva ou de emergência, podem trazer sintomas, principalmente a dispareunia, nos primeiros meses após o parto.

No estudo de Amiri et al., 2017, realizado no Irã, houve semelhanças com os outros estudos citados, onde foi realizado um estudo transversal com mulheres em duas etapas; gravidez precoce e 3 a 6 meses após o parto em centros de saúde. Os resultados sexuais femininos foram avaliados através do Índice de Função Sexual Feminina e o tempo necessário para retomar atividades sexuais após o parto. A função sexual não diferiu significativamente entre os dois grupos de parto vaginal $n=90$ e cesariana $n=113$ em relação à duração do casamento, nível educacional, métodos anticoncepcionais e status ocupacional. Relacionado ao tempo médio de a retomada da atividade sexual não houve conflito substancial entre os dois grupos. A frequência média de relações sexuais no período pós-parto foi de 1,8 $\pm 1,2$ vezes por semana, com diferença significativa em comparação com pré-gravidez. As pontuações dos domínios individuais após o parto foram significativamente mais baixas em comparação com a pré-gravidez. Concluindo que não há diferenças nos resultados sexuais entre parto vaginal e cesariana.

Fan et al. 2017, desempenharam pesquisas eletrônicas, na China, em diversas bases de dados e notaram que o desfecho primário foi a satisfação sexual e os secundários foram retomadas da relação sexual e dor sexual no pós-parto. Esta meta-análise indicou que a via de parto cesárea e vaginal não afetou a satisfação sexual pós-parto e pareceu ter efeito mínimo sobre as relações sexuais após uma retomada com um período maior.

Dean et al. 2008, corroboraram com o estudo de Pereira et al., 2018, Moura et al., 2018, Amiri et al., 2017 e Fan et al., 2017. este foi um inquérito postal transversal de mulheres, seis anos após o parto, que deram à luz em maternidades em Aberdeen, Birmingham e Dunedin e responderam a um questionário anterior em seis anos após o parto indexado, 4.214 mulheres responderam, das quais 2.765 (65\%) responderam dez questões de função sexual. Embora tenha havido pouca associação entre o histórico do modo de parto e a maioria das questões de função sexual, mulheres que tiveram parto exclusivamente por cesariana tiveram pontuação significativamente melhor nas questões relacionadas à sua percepção do tônus vaginal por avaliação delas próprias e do parceiro na satisfação sexual, especialmente quando comparada com mulheres que tiveram partos instrumentais. Mulheres que relataram que estavam realizando exercícios para o assoalho pélvico tiveram uma pontuação significativa melhor em sete questões. Mulheres com incontinência urinária ou fecal pontuaram significativamente mais baixo em todos os aspectos sexuais nas questões de função. A história do tipo de parto pareceu ter efeito mínimo sobre a função sexual.

O estudo de Anglès-Acedo et al., 2019, não corroborou com os demais estudos, onde, na Espanha, 318 mulheres foram incluídas no estudo sendo que 140 mulheres com histórico de lesões obstétricas no esfíncter anal e 178 mulheres sem lesões. Diferenças importantes foram observadas relacionadas ao modo de parto, depois de um parto vaginal operatório, o grupo com lesões parecia retomar a atividade sexual antes do grupo sem lesões (60\% vs. 78\%). Após um parto espontâneo, os pacientes sem lesões apresentaram uma porcentagem maior de retomada na relação sexual do que aqueles com lesões (98\% vs. 77\%). A história da lesão obstétrica atrasa a retomada de atividade sexual entre mulheres com parto espontâneo. No entanto, um histórico de parto vaginal operatório parece ter um maior impacto nessa decisão, refletindo assim os efeitos de longo prazo de graves traumas perineais e pélvicos na sexualidade pós-parto.

No estudo de Baud et al., 2020, realizado na Suíça, concordou com o estudo de Anglés-Acedo demonstrando diferenças nas vias de parto, as mulheres que mantiveram parto cesáreo eletivo único relataram significativamente mais dificuldades relacionadas a lubrificação e satisfação sexual, do que as mulheres de partos vaginais sem complicações (36,2\% versus $26,4 \%$, respectivamente). Todos os itens que investigam a dor durante ou após a relação sexual foram significativamente piores após parto cesáreo eletivo único do que partos vaginais sem complicações. Nenhuma das outras pontuações, para excitação ou orgasmo mostrou diferenças significativas. Dispareunia foram significativamente mais 
frequentes após parto cesáreo eletivo único do que parto vaginal sem complicações.

No estudo de Hjorth et al., 2019, realizado na Dinamarca, Inglaterra e Noruega, foi analisado o questionário de 36.691 mulheres sobre necessidades sexuais e observaram que 38\% destas relataram um ou mais problemas como: desejo sexual reduzido ou ausente, onde em alguns casos (35\%) afetava o seu parceiro. A frequência de problemas sexuais entre as mulheres apenas com partos vaginais espontâneos, foi de 37\%. Para as mulheres que só tiveram cesarianas, mais problemas foram relatados. Já aquelas que tiveram parto vaginal espontâneo subsequente a uma cesariana, e para mulheres apenas com partos vaginais que tiveram um ou mais partos vaginais instrumentais, as chances de problemas sexuais não diferiram das mulheres com apenas partos vaginais espontâneos. Portanto, o parto vaginal parece estar associado a menos problemas sexuais, mesmo quando envolve parto instrumental ou episiotomia.

\section{- Laceração/trauma perineal e suas implicações nas DS no pós-parto.}

Anglès-Acedo et al. 2019, estudaram a retomada do coito pós-parto em mulheres com e sem lesão obstétrica do esfíncter anal (OASIS), na Espanha, e observaram que a retomada do coito em 6 meses foi de $73 \%$, sem diferenças estatisticamente significativas entre os grupos OASIS vs. não OASIS. No entanto, Rogers et al., 2009, analisaram 576 mulheres americanas que tiveram parto vaginal com trauma espontâneo do trato genital constataram que aproximadamente metade das mulheres havia retomado a atividade sexual por volta de 6 semanas após o parto.

Lawrence et al., 2016, analisaram o efeito das lacerações em 448 americanas que realizaram parto vaginal. Destas $33,7 \%$ sofreram trauma perineal sendo destes $85 \%$ classificada em laceração de segundo grau e $2 \%$ em quarto grau. Contudo, na pesquisa de Rogers et al., 2009, a maioria das mulheres sexualmente ativas sofreram trauma perineal sendo apenas $16 \%$ com o trato genital intacto após o parto.

Além do mais, Lawrence et al., 2016, observaram algumas variáveis nas mulheres com trauma perineal, como: eram mais velhas (25. vs. 23,$0 ; p<0,001)$, tinham mais anos de escolaridade (14,9 vs. 13,$3 ; \mathrm{p}<0,001)$ ), eram mais altas $(64,7$ vs. $63,7 \mathrm{~cm} ; \mathrm{p}<0,001)$, Mulheres americanas hispânicas eram mais propensas e um maior peso médio ao nascer e ter um bebê pequeno para a idade gestacional (PIG) foi protetor. Das variáveis de parto, obteve-se que a duração do segundo estágio ativo (95 vs. 613 minutos; p <0,0001) e um bebê partindo occipital posterior ou transversal $(6,0 \%$ vs. 1,6\%; p =0,02) foram significativos. Corroborando com Rogers et al., 2009, que também observaram que mulheres com traumas graves completaram mais anos de escolaridade, estiveram ativamente no trabalho de parto por mais tempo. 13\% (36/276) das mulheres tiveram trauma perineal que exigiu sutura. Mulheres com traumas suturados eram menos propensos a serem sexualmente ativas do que mulheres sem traumas suturados (36/50 (72\%) vs. 240/276 (87\%) sexualmente ativas, P = 0,01). Gommesen et al., 2019, examinaram a associação entre o grau de laceração perineal e a função sexual 12 meses após o parto em 554 Dinamarquesas através do PISQ-12 e um exame clínico. As mulheres que sofreram lacerações de $3^{\circ}$ ou $4^{\circ}$ grau eram em média 0,5 anos mais velhas do que as mulheres com lacerações de $2^{\circ}$ grau e 1,2 anos mais velhas do que as mulheres que não apresentavam lacerações de primeiro grau. Complementando, Gommesen et al., 2019, observaram maior ruptura com maior peso ao nascer, segundo estágio mais longo do trabalho de parto e maior duração do parto ativo. O parto instrumental foi mais frequente entre mulheres com lacerações de segundo grau $(15 \%)$ e de $3^{\circ}$ e $4^{\circ}$ grau (34\%) em comparação com mulheres sem lacerações ou $1^{\circ}$ $(3 \%)$.

Lawrence et al., 2016, observaram que o grupo que deu à luz com períneo intacto ou trauma menor teve função sexual pré-natal mais alta com base nos escores do Índice de Função Sexual Feminina $(26,9$ vs 25,3; p = 0,03) e teve maior probabilidade de ter sido sexualmente ativo no terceiro trimestre $(82,4$ vs $65,1 \% ; p<0,001)$. Já mulheres com trauma perineal tiveram pontuações mais baixas no Índice de Função Sexual Feminina, indicando pior função sexual $(27,3$ vs 29,1; p = 0,01), principalmente nos domínios de excitação, dor e satisfação. Corroborando com o estudo de Rogers et al., 2009, as mulheres 
sexualmente ativas com trauma grave tiveram pontuações do Intimate Relationship Scale (IRS) mais baixas do que mulheres com trauma menor, $(33,5+/-6,4$ vs $35,6+/-8,0$ pontuações totais de IRS, $\mathrm{p}=0,02)$. Esta relação foi mais forte para o subconjunto de mulheres que se submeteram à sutura perineal versus aquelas que não o fizeram $(31,8+/-6,7$ vs $35,5+/$ - 7,6 pontuações totais de IRS, $P$ = 0,007). Além disso, Gommesen et al., 2019, descobriram que mulheres fumantes têm uma pontuação PISQ-12 mais alta em comparação com mulheres não fumantes ( $\beta$ 2,99 ajustados; IC 95\% 0,93 a 5,06).

Lawrence et al., 2016, identificaram que mulheres com trauma perineal profundo relataram dor perineal em 6 meses do que mulheres com trauma menos grave $(15,5$ vs. $6,2 \%$; $=0,01)$, no entanto, a intensidade da dor não foi diferente entre os grupos $(0,38$ vs. 0,$12 ; \mathrm{p}=0,19)$. Quando mulheres com lacerações de $3^{\circ}$ e $4^{\circ}$ graus foram excluídas do grupo de trauma profundo, os achados de uma maior proporção de mulheres com dor aos 6 meses $(17,8 \%$ vs $6,2 \%, p=0,04)$ ainda estavam presentes. No entanto, Rogers et al., 2009, perceberam na análise univariada, que as mulheres com traumas maiores eram mais propensas a relatar que a fadiga interferia no ato sexual, menos satisfação com as atividades sexuais, menos desejo de ser abraçada, tocada e acariciada e menos desejo de relações sexuais. Mulheres com traumas graves não eram mais propensas do que mulheres com traumas menores a relatar "mais ou muito mais dor" com a relação sexual (24 (38\%) vs $40(63 \%), p=0,16)$ ou "menos ou muito menos" capacidade de atingir o orgasmo, (30/83 (36\%) vs 47/144 (24\%), p = 0,06), mas relatou "menos ou muito menos" satisfação com sua aparência corporal $(55 / 63$ (66\%) vs 88/189 $(47 \%), p=0,003)$.

O’Malley et al., 2018, pesquisaram fatores de risco para problemas sexuais aos 6 e 12 meses pós-parto em 832 mulheres Irlandesas. Em comparação com mulheres com períneo intacto, mulheres que tiveram rupturas perineais de $2^{\circ}$ grau (OR 1,6, IC 95\% 1,0-2,3), episiotomia (OR 1,7, IC 95\% 1,2-2,5) ou lacerações de $3^{\circ}$ grau (OR 3,7, 95\% IC 1,5-9,3), foram significativamente mais propensos a experimentar dispareunia 6 meses após o parto, o que persistiu por 12 meses tanto para episiotomias quanto para lacerações perineais de $3^{\circ}$ grau. Amamentação e laceração perineal de $3^{\circ}$ grau foram ambos associados a experimentar dispareunia 6 meses após nascimento. Para Gommesen et al., 2019, a proporção de dispareunia prégravidez foi de $14 \%, 21 \%$ e $24 \%$ nos grupos de $1^{\circ}, 2^{\circ}$ e $3^{\circ}$ ou $4^{\circ}$, respectivamente. Aos 12 meses após o parto, a proporção em todos os três grupos era maior do que antes da gravidez, $25 \%, 38 \%$ e $53 \%$, respectivamente. Em comparação mulheres sem ou com lacerações de primeiro grau com as que tiveram lacerações de terceiro ou quarto grau tiveram um risco maior de dispareunia (RR ajustado (aRR) 2,09; IC de 95\% 1,55 a 2,81).

\section{- Intervenções Fisioterapêuticas nas DS no puerpério.}

Dean et al., 2008, investigaram a relação da função sexual com exercícios do assoalho pélvico para incontinência em 2675 puérperas da Inglaterra, Escócia e Nova Zelândia. As mulheres em treinamento pontuaram significativamente melhor em sete das dez perguntas principalmente as que estavam relacionadas com dor durante a relação sexual, incontinência urinária e tônus vaginal para a satisfação de seu parceiro. Contudo, na revisão sistemática de Hadizadeh-Talasaz, Sadeghi e Khadivzadeh 2019 sobre efeito do exercício do assoalho pélvico na função sexual pós-parto evidenciaram que o treinamento da musculatura do assoalho pélvico em mulheres primíparas ou multíparas pode aumentar a função sexual e a qualidade de vida no pós-parto.

Porém Huang et al., 2019 avaliaram um outro tipo de intervenção na dor perineal pós-parto e função sexual em 78 taiwanesas primíparas submetidas a episiotomia e laceração de $2^{\circ}$ grau utilizando da radiação infravermelha e concluíram que a maioria das mulheres teve dor perineal leve uma semana após o parto $(1,1 \pm 0,9$ no grupo controle vs $1,4 \pm 1,5$ no grupo FIR) e o grupo intervenção mostrou uma pontuação mais alta de melhora na dor perineal imediatamente após o parto e uma semana após o parto no (diferença de pontuação: 5,2), mas nenhuma diferença significativa em comparação com o grupo de controle. Em relação a função sexual o grupo intervenção teve uma pontuação total PISQ-12 mais alta em 3 meses e 6 meses após o parto, indicando melhor função sexual em comparação com o grupo de controle, mas não houve diferença estatisticamente significativa. Quando questionados sobre a presença de dor durante a relação sexual, os participantes do grupo controle tiveram 
menos dor, mas não houve diferença significativa entre os grupos.

\section{Conclusão}

Conclui-se que as principais causas das disfunções sexuais foram a ansiedade e depressão, principalmente no puerpério imediato, onde se observou uma necessidade de compreensão do parceiro para a retomada das atividades sexuais. Ademais, a literatura não apresenta consenso sobre qual via de parto sugere mais disfunções sexuais, contudo, se orienta que as mulheres primíparas devem ter mais cautela na escolha da via de parto de maneira a prevenir complicações posteriores.

Salienta-se que o histórico de lesão obstétrica pélvica atrapalha o retorno das atividades sexuais, gerando mais dor e fadiga durante o coito. A via de parto cesárea, por sua vez, diminui a lubrificação e satisfação sexual. Como forma de reabilitação, observa-se a importância do tratamento da dor perineal de maneira precoce, bem como o treino do assoalho pélvico para o retorno da vida sexual sem queixas.

Como limitações da pesquisa, foram encontrados poucos artigos que discutissem sobre a interferência de fatores psicossociais na disfunção sexual e de métodos intervencionistas fisioterapêuticos para tratar tais afecções. Além da necessidade de análise dos estudos por um período prolongado. Portanto, sugere-se continuação de pesquisas podendo abordar especificamente cada um dos fatores encontrados através desta revisão sistemática, com melhor rigor metodológico para que seja esclarecido a melhor escolha do parto com menos riscos de disfunções sexuais no puerpério, sendo trabalhos detalhados e claros, que incluam uma padronização, contribuindo para a prática baseada em evidências e melhoria da qualidade de vida das puérperas.

\section{Agradecimentos}

Agradecemos a todos que direta e indiretamente contribuíram para a realização e sucesso do artigo.

\section{Referências}

Amiri, F. N., Omidvar, S., Bakhtiari, A., \& Hajiahmadi, M. (2017). Female sexual outcomes in primiparous women after vaginal delivery and cesarean section. African health sciences, 17(3), 623-631.

Anglès-Acedo, S., Ros-Cerro, C., Escura-Sancho, S., Elías-Santo-Domingo, N., Palau-Pascual, M. J., \& Espuña-Pons, M. (2019). Coital resumption after delivery among OASIS patients: differences between instrumental and spontaneous delivery. BMC women's health, 19(1), 1-7.

Baud, D., Sichitiu, J., Lombardi, V., De Rham, M., Meyer, S., Vial, Y., \& Achtari, C. (2020). Comparison of pelvic floor dysfunction 6 years after uncomplicated vaginal versus elective cesarean deliveries: a cross-sectional study. Scientific Reports, 10(1), 1-8.

Brasil, Ministério da Saúde. Banco de dados do Sistema Único de Saúde-

Cardoso, P. O., Alberti, L. R., \& Petroianu, A. (2010). Morbidade neonatal e maternas relacionada ao tipo de parto. Ciência \& Saúde Coletiva, 15 , 427-435.

Chaparro, M.; Pérez, R.; \& Sáez, K. Función sexual femenina durante el período posparto. Revista de Obstetricia y Ginecología de Venezuela, 73(3), 181-186, 2013 .

DATASUS, Sistema de Informações Hospitalares. http://www.datasus.gov.br/catalogo/sihsus.htm, 2009.

de MOURA, T. R., Nunes, E. F. C., sutter Latorre, G. F., \& Vargas, M. M. (2018). Dispareunia relacionada à via de parto: uma revisão integrativa. Revista de Ciências Médicas, 27(3), 157-165.

Dean, N., Wilson, D., Herbison, P., Glazener, C., Aung, T., \& MacArthur, C. (2008). Sexual function, delivery mode history, pelvic floor muscle exercises and incontinence: A cross-sectional study six years post-partum. Australian and New Zealand Journal of Obstetrics and Gynaecology, $48(3), 302-311$.

Faisal-Cury, A., Huang, H., Chan, Y. F., \& Menezes, P. R. (2013). The relationship between depressive/anxiety symptoms during pregnancy/postpartum and sexual life decline after delivery. The journal of sexual medicine, 10(5), 1343-1349.

Fan, D., Li, S., Wang, W., Tian, G., Liu, L., Wu, S., \& Liu, Z. (2017). Sexual dysfunction and mode of delivery in Chinese primiparous women: a systematic review and meta-analysis. BMC pregnancy and childbirth, 17(1), 1-9.

Galvão, M. C. B., \& Ricarte, I. L. M. (2019). Revisão sistemática da literatura: conceituação, produção e publicação. Logeion: Filosofia da informação, 6(1), 57-73. 
Research, Society and Development, v. 11, n. 2, e23111225638, 2022

(CC BY 4.0) | ISSN 2525-3409 | DOI: http://dx.doi.org/10.33448/rsd-v11i2.25638

Girardi, F. (2019). Itinerários de cuidado e práticas de atenção à saúde das mulheres kaingang no período gravídico-puerperal na aldeia Kondá/SC.

Gommesen, D., Nøhr, E., Qvist, N., \& Rasch, V. (2019). Obstetric perineal tears, sexual function and dyspareunia among primiparous women 12 months postpartum: a prospective cohort study. BMJ open, 9(12), e032368.

Hadizadeh-Talasaz, Z., Sadeghi, R., \& Khadivzadeh, T. (2019). Effect of pelvic floor muscle training on postpartum sexual function and quality of life: A systematic review and meta-analysis of clinical trials. Taiwanese Journal of Obstetrics and Gynecology, 58(6), 737-747.

Hjorth, S., Kirkegaard, H., Olsen, J., Thornton, J. G., \& Nohr, E. A. (2019). Mode of birth and long-term sexual health: a follow-up study of mothers in the Danish National Birth Cohort. BMJ open, 9(11), e029517.

Huang, L. H., Lai, Y. F., Chen, G. D., Lee, M. S., \& Ng, S. C. (2019). Effect of far-infrared radiation on perineal wound pain and sexual function in primiparous women undergoing an episiotomy. Taiwanese Journal of Obstetrics and Gynecology, 58(1), 68-71.

Lawrence, L., Rebecca, R., Noelle, B., Dusty, T., \& Clifford, Q. (2016). The effect of perineal lacerations on pelvic floor function and anatomy at six months postpartum in a prospective cohort of nulliparous women. Birth (Berkeley, Calif.), 43(4), 293.

Mascarello, K. C., Matijasevich, A., Santos, I. D. S. D., \& Silveira, M. F. (2018). Early and late puerperal complications associated with the mode of delivery in a cohort in Brazil. Revista Brasileira de Epidemiologia, 21.

O'Malley, D., Higgins, A., Begley, C., Daly, D., \& Smith, V. (2018). Prevalence of and risk factors associated with sexual health issues in primiparous women at 6 and 12 months postpartum; a longitudinal prospective cohort study (the MAMMI study). BMC pregnancy and childbirth, 18(1), 1-13.

Pereira, T. R. C., Dottori, E. H., Mendonça, F. M. D. A. F., \& Beleza, A. C. S. (2018). Avaliação da função sexual feminina no puerpério remoto: um estudo transversal. Revista Brasileira de Saúde Materno Infantil, 18, 289-294.

Pereira, T. R. C., Dottori, E. H., Mendonça, F. M. D. A. F., \& Beleza, A. C. S. (2018). Avaliação da função sexual feminina no puerpério remoto: um estudo transversal. Revista Brasileira de Saúde Materno Infantil, 18, 289-294.

Rogers, R. G., Borders, N., Leeman, L. M., \& Albers, L. L. (2009). Does spontaneous genital tract trauma impact postpartum sexual function? Journal of midwifery \& women's health, 54(2), 98-103.

Santana, L. S., Gallo, R. B. S., Marcolin, A. C., Ferreira, C. H. J., \& Quintana, S. M. (2011). Utilização dos recursos fisioterapêuticos no puerpério: revisão da literatura. Femina. 